\title{
A set oriented approach to optimal feedback stabilization
}

\author{
Lars Grüne \\ Mathematisches Institut, Universität Bayreuth, 95440 Bayreuth, Germany \\ Oliver Junge* \\ Institut für Mathematik, Universität Paderborn, 33095 Paderborn, Germany
}

\begin{abstract}
We present a numerical construction of an optimal control based feedback law for the stabilization of discrete time nonlinear control systems. The feedback is based on a recently developed numerical solution method for optimal control problems using set oriented and graph theoretic algorithms. We show how this method can be used to construct approximately optimal and stabilizing feedback laws and present an a posteriori error estimation technique for the adaptive generation of the underlying set oriented space discretization.
\end{abstract}

Key words: feedback stabilization; nonlinear control system; optimal control; set oriented numerics; shortest path

\section{Introduction}

Optimization based stabilization has been a popular method for many decades, in particular for linear systems where the linear quadratic controller design is a by now classical method, see (Sontag, 1998, Chapter 8). The key property that makes this method work is an appropriately chosen infinite horizon cost functional which will guarantee that an optimal feedback law is also a stabilizing controller.

* corresponding author, phone +495251602642, fax +495251604216

Email addresses: lars.gruene@uni-bayreuth.de (Lars Grüne), junge@upb.de (Oliver Junge). 
For nonlinear systems stabilization techniques based on direct applications of infinite horizon optimal control are much less popular. The reason lies in the fact that nonlinear infinite horizon optimal control problems are difficult to solve, both analytically and numerically. Hence, instead of solving such problems, one often tries to circumvent the difficulties, as, e.g., in inverse optimal design where first a suitable Lyapunov function is designed by techniques like backstepping and then an optimal control is identified for which this function is the optimal value function, see e.g. Freeman and Kokotovic (1996); Freeman and Kokotović (1996). On the numerical side a popular way to avoid the inherent difficulties of infinite horizon optimal control is receding horizon or model predictive control where the solutions of a sequence of finite horizon optimal control problems are used for the feedback design, see e.g. Fontes (2001); Mayne et al. (2000) and the references therein.

Theoretically, however, infinite horizon optimal control based design is very attractive provided the corresponding optimization problem is numerically feasible, because it is flexible due to the choice of the cost function, does not need any analytical a priori information like Lyapunov functions (in contrast to the inverse optimal approach), allows for the treatment of deterministic and stochastic perturbations and - since the main numerical work is done offline - provides feedback laws whose numerical evaluation is fast and easy to implement (in contrast to the receding horizon approach). Approaches for the construction of such feedback laws reported in the literature (see e.g. Kreisselmeier and Birkhölzer (1994), Grüne and Wirth (1999) or Grüne (2000)) demonstrate that this method can work very well, furthermore, a theoretical framework for the rigorous treatment of sampled data systems using this approach has recently been developed Grüne and Nešić (2003).

In the present paper, we follow this infinite horizon approach for discrete time nonlinear systems using a recently developed set oriented technique for the numerical solution of global optimal control problems from Junge and Osinga (2004). Set oriented numerical methods have been developed for the analysis of invariant objects for dynamical and control systems, see Dellnitz and Hohmann (1997); Dellnitz and Junge (2002); Szolnoki (2003) and also (Grüne, 2002, Chapters 6 and 7). In these methods the state space is divided into cells and the underlying dynamics of the control system is represented by a graph on the set of cells. The combination of hierarchical tree data structures with subdivision techniques which allow to adaptively refine those parts of the state space where high accuracy is needed make these methods very appealing and highly efficient. The connection to optimal control problems was established in Junge and Osinga (2004) via the observation that the graph on the cells can be complemented by suitably chosen weights on the edges representing the cost function which then allows for the use of fast graph theoretic algorithms for the solution of the numerical optimization problem. 
While the convergence of this method in terms of the optimal value function was proved in Junge and Osinga (2004) it was not completely clear whether and how one can use this approximate solution for the feedback design problem. Our paper closes this gap by combining ideas from set oriented methods Junge and Osinga (2004), dynamic programming Grüne (1997) and Lyapunov based approximate stability analysis Nešić and Teel (2004) in order to construct a suitable feedback law and prove approximate asymptotic stability for the resulting closed system. In addition, we develop an error estimation technique which is adapted to the stabilization problem and allows to derive an adaptive subdivision of the cell covering used in our numerical approximation.

The paper is organized as follows. In the ensuing Section 2 we describe the problem formulation in detail. In Section 3 we explain the set oriented numerical solution from Junge and Osinga (2004). The construction of our feedback is presented in Section 4 together with a proof of approximate optimality for the case of equally sized cells. The error estimation and adaptive subdivision ideas and the corresponding optimality and stability proof for the resulting feedback law are given in Section 5 and, finally, two numerical examples are presented in Section 6.

\section{Problem formulation}

We consider the problem of optimally stabilizing the discrete-time control system

$$
x_{k+1}=f\left(x_{k}, u_{k}\right), \quad k=0,1, \ldots,
$$

subject to the instantaneous cost $g: X \times U \rightarrow[0, \infty)$. Here $f: X \times U \rightarrow \mathbb{R}^{d}$ is continuous, $f(0,0)=0, X \subset \mathbb{R}^{d}, 0 \in X$, is compact, $U \subset \mathbb{R}^{m}, 0 \in U$, is compact and $g(x, u)>0$ for all $x \neq 0$. Let $\mathcal{U}(x)=\left\{\mathbf{u} \in U^{\mathbb{N}}: x_{k}(x, \mathbf{u}) \rightarrow\right.$ 0 as $k \rightarrow \infty\}$ the set of stabilizing control sequences for $x \in X$ and $S=\{x \in$ $X: \mathcal{U}(x) \neq \emptyset\}$ the stabilizable subset $S \subset X$. The total cost along a controlled trajectory is given by

$$
J(x, \mathbf{u})=\sum_{k=0}^{\infty} g\left(x_{k}(x, \mathbf{u}), u_{k}\right) \in[0, \infty]
$$

and we assume that $g$ is chosen such that this sum is finite for each $x \in S$ and appropriately chosen $\mathbf{u}$ (see Grüne and Nešić (2003) for details on how such a $g$ can be found).

Our goal is to construct an approximate optimal feedback $u: S \rightarrow U$ such that we can prove a suitable approximate asymptotic stability property for the resulting closed loop system. The construction will be based on an ap- 
proximation of the (optimal) value function $V: S \rightarrow[0, \infty]$,

$$
V(x)=\inf _{\mathbf{u} \in \mathcal{U}(\mathbf{x})} J(x, \mathbf{u})
$$

which will act as a Lyapunov function in the asymptotic stability proofs.

\section{Computational approach}

In this section we discuss a set oriented numerical method for the computation of $V$ which was developed in Junge and Osinga (2004). The method relies on the observation that one may formulate the above discrete-time optimal control problem equivalently as the problem of finding a shortest path within a directed weighted graph $G=(X, E)$, where the set $E$ of edges of $G$ is given by all pairs $(x, f(x, u)), x \in X, u \in U$, and the weight $w(e) \in[0, \infty)$ of the edge $e=(x, f(x, u))$ is given by $w(e)=g(x, u)$.

We roughly touch on this equivalent formulation, because we are going to use a finite version of the graph $G$ in order to compute an approximation to $V$. The idea is that on a finite graph which in a certain sense is an approximation to $G$, we can apply standard algorithms for computing shortest paths. More precisely, the algorithm that we need here computes "all source, single destination shortest paths", since we are interested in computing the cost of the optimal trajectory for all initial conditions, but for a single target point. A typical algorithm of this type is Dijkstra's algorithm Dijkstra (1959). In a suitable implementation this algorithm has complexity $\mathcal{O}((|X|+|E|) \log |X|)$, where $|X|$ is the number of nodes and $|E|$ is the number of edges of the graph $G$.

The finite approximation to $G$ is constructed as follows: Let $\mathcal{P}$ be a partition of $X$, that is, a finite collection of compact subsets $P_{i} \subset X, i=1, \ldots, r$, with $\cup_{i=1}^{r} P_{i}=X$, and $m\left(P_{i} \cap P_{j}\right)=0$ for $i \neq j$ (where $m$ denotes Lebesgue measure). Define the graph

$$
G_{\mathcal{P}}=\left(\mathcal{P}, E_{\mathcal{P}}\right), \quad E_{\mathcal{P}}=\left\{\left(P_{i}, P_{j}\right) \in \mathcal{P} \times \mathcal{P} \mid f\left(P_{i}, U\right) \cap P_{j} \neq \emptyset\right\},
$$

where the edge $e=\left(P_{i}, P_{j}\right)$ carries the weight

$$
w(e)=\min _{x \in P_{i}, u \in U}\left\{g(x, u) \mid f(x, u) \in P_{j}\right\} .
$$

We use $G_{\mathcal{P}}$ to find an approximation to the optimal value function $V$. For any $x \in X$ there is a least one subset $P \in \mathcal{P}$ containing $x$. The approximation for $V(x)$ will be the length $w(p)$ of a shortest path $p$ from a node $P, x \in P$, to a 
node $P^{\prime} \in \mathcal{P}$ that contains the origin, i.e. we approximate $V(x)$ by

$$
V_{\mathcal{P}}(x)=\min \left\{w(p) \mid p \text { is a path from a set } P, x \in P \text {, to a set } P^{\prime}, 0 \in P^{\prime}\right\} .
$$

3.0.0.1 Convergence. Let $(\mathcal{P}(l))_{l}$ be a nested sequence of partitions of $X$ (i.e. for every $l$, each element of $\mathcal{P}(l+1)$ is contained in an element of $\mathcal{P}(l)$ ). One can show that for $x \in S, V_{\mathcal{P}(l)}$ converges pointwise to $V$, see Junge and Osinga (2004). Here we additionally show the following

Proposition 1 Let $D \subset S$ be a set on which $V$ is continuous. Then $V_{\mathcal{P}(l)} \rightarrow V$ uniformly on every closed subset $\bar{D} \subset D$.

Proof. Let $\varepsilon>0$. By continuity of $V$ and compactness of $\bar{D}$, we can choose a finite subset $C \subset \bar{D}$ and compact neighborhoods $B_{x} \subset \bar{D}, x \in C$, such that $\bar{D}$ is contained in the union of the $B_{x}, x \in C$, and $|V(y)-V(x)| \leq \varepsilon / 2$ for all $y \in B_{x}$.

Consider $B_{x}$ for $x \in C$. Since $V_{\mathcal{P}(l)}$ converges pointwise to $V$ on $D$, for every $y \in B_{x}$ there is an $l_{y}$ such that $V(y)-V_{\mathcal{P}(l)}(y) \leq \varepsilon / 2$ for all $l \geq l_{y}$. What is more, since $V_{\mathcal{P}(l)}$ is piecewise constant, for every $y \in B_{x}$ and every $l \in \mathbb{N}$ there is an open neighborhood $N_{y}(l) \subset B_{x}$ of $y$, such that $V_{\mathcal{P}(l)}(z) \geq V_{\mathcal{P}(l)}(y)$ for all $z \in N_{y}(l):$

- Either $y$ is in the interior of some partition element $P \in \mathcal{P}(l)$. Since $V_{\mathcal{P}(l)}$ is constant on $\operatorname{int}(P)$, there is a neighborhood $N_{y}(l) \subset P$ of $y$ such that $V_{\mathcal{P}(l)}(z)=V_{\mathcal{P}(l)}(y)$ for all $z \in N_{y}(l)$

- Or $y$ is on the boundary of several partition elements. Then, by definition of $V_{\mathcal{P}(l)}$,

$$
V_{\mathcal{P}(l)}(y)=\min \left\{V_{\mathcal{P}(l)}(z): z \in P, y \in P, P \in \mathcal{P}(l)\right\},
$$

and there is a neighborhood $N_{y}(l) \subset \bigcup_{y \in P, P \in \mathcal{P}(l)} P$ such that $V_{\mathcal{P}(l)}(z) \geq$ $V_{\mathcal{P}(l)}(y)$ for all $z \in N_{y}(l)$.

The collection $N_{y}\left(l_{y}\right), y \in B_{x}$, is an open covering of $B_{x}$ and since $B_{x}$ is compact, we can choose a finite subset $A_{x} \subset B_{x}$, such that the collection $N_{y}\left(l_{y}\right), y \in A_{x}$, still covers $B_{x}$. Let $l_{x}=\max _{y \in A_{x}} l_{y}$. Since $V_{\mathcal{P}(l)}$ is monotonously increasing, we have that

$$
V_{\mathcal{P}(l)}(z) \geq V_{\mathcal{P}\left(l_{y}\right)}(y) \quad \text { for all } z \in N_{y}\left(l_{y}\right) \text { and all } l \geq l_{y},
$$

so in particular $V(y)-V_{\mathcal{P}(l)}(y) \leq \varepsilon / 2$ for all $y \in B_{x}$ and all $l \geq l_{x}$.

Choosing $L=\max _{x \in C} l_{x}$ we get that $V(x)-V_{\mathcal{P}(l)}(x) \leq \varepsilon / 2$ for all $x \in \bar{D}$ and all $l \geq L$, which completes the proof. 
3.0.0.2 Implementation. The computation of $V_{\mathcal{P}}$ breaks down into three steps:

(1) Construction of a suitable partition $\mathcal{P}$;

(2) Construction of $G_{\mathcal{P}}$;

(3) Computation of $V_{\mathcal{P}}$ by applying Dijkstra's algorithm to $G_{\mathcal{P}}$.

In the numerical realization we always let $X$ be a box in $\mathbb{R}^{d}$ and construct a partition of $X$ by dividing $X$ uniformly into smaller boxes. In fact, we realize this division by repeatedly bisecting the current division (changing the coordinate direction after each bisection). The resulting sequence of partitions can efficiently be stored as a binary tree — see Dellnitz and Hohmann (1997) for more details.

Once $\mathcal{P}$ has been constructed, we need to compute the set $E_{\mathcal{P}}$ of edges of $G_{\mathcal{P}}$, as well as the weight $w(e)$ for every edge $e \in E_{\mathcal{P}}$. Naively, one is faced with $r^{2}$ optimization problems (where $r$ is the number of elements in the partition $\mathcal{P})$ - one for each pair $\left(P_{i}, P_{j}\right)$ of partition elements. The objective function is $g$, and for the pair $\left(P_{i}, P_{j}\right)$, the constraints on $x$ and $u$ are given by the requirements $f(x, u) \in P_{j}, x \in P_{i}$.

However, using the hierarchical construction of the partitions, one can reduce the complexity of the problem. Here we simply use the following approach: we approximate $E_{\mathcal{P}}$ by

$$
\tilde{E}_{\mathcal{P}}=\left\{\left(P_{i}, P_{j}\right) \mid f\left(\tilde{P}_{i}, \tilde{U}\right) \cap P_{j} \neq \emptyset\right\},
$$

where $\tilde{P}_{i} \subset P_{i}$ and $\tilde{U} \subset U$ are finite sets of "test points". For example, one may choose these sets as points on an equidistant grid. Correspondingly the weight $w(e)$ on $e=\left(P_{i}, P_{j}\right)$ is approximated by

$$
\tilde{w}(e)=\min _{x \in \tilde{P}_{i}, u \in \tilde{U}}\left\{g(x, u) \mid f(x, u) \in P_{j}\right\} .
$$

Again, we refer to Dellnitz and Hohmann (1997) and Junge and Osinga (2004) for further details.

\section{Constructing the feedback}

It was shown in Junge and Osinga (2004) that the set oriented algorithm gives approximately optimal pseudo-trajectories by following the shortest path on the partitions linking the initial value to the subset $P_{0} \in \mathcal{P}$ containing the origin. Here the term pseudo-trajectory refers to the fact that at each time instance a small jump (corresponding to the size of the current cell $P$ ) in the 
trajectory is introduced. It is by no means clear how and under which conditions these pseudo-trajectories can be used for the generation of approximate optimal real trajectories.

Hence, our construction of the approximately optimal feedback law uses a different idea, namely the classical dynamic programming technique. It follows from standard dynamic programming arguments that the exact optimal value function $V$ satisfies

$$
V(x)=\inf _{u \in U}\{g(x, u)+V(f(x, u))\}
$$

and that an optimal feedback law $u$ is given by the control $u(x)$ minimizing the right hand side of this equation, provided this minimum exists.

For the construction of our feedback law we will use this fact, replacing $V$ by its approximation $V_{\mathcal{P}}$. Thus for each point $x \in S$ we define

$$
u_{\mathcal{P}}(x)=\operatorname{argmin}_{u \in U}\left\{g(x, u)+V_{\mathcal{P}}(f(x, u))\right\}
$$

Note that the minimum exists because $V_{\mathcal{P}}$ admits only finitely many values.

The following theorem shows in which sense this feedback is approximately optimal.

Theorem 2 Consider a sequence of partitions $\mathcal{P}(l), l \in \mathbb{N}$ and let $D \subseteq S$ be an open set with the following properties.

(i) $0 \in \operatorname{int} D$

(ii) $\mathcal{U}(x) \neq \emptyset$ for all $x \in D$

(iii) For each $\varepsilon>0$ there exists $l_{0}(\varepsilon)>0$ such that that the inequality

$$
V(x)-V_{\mathcal{P}(l)}(x) \leq \varepsilon
$$

holds for all $x \in D$ and all $l \geq l_{0}(\varepsilon)$.

Let $c>0$ be the largest value such that the inclusion $D_{c}(l):=V_{\mathcal{P}(l)}^{-1}([0, c]) \subset D$ holds for all $l \in \mathbb{N}$.

Then there exists $\varepsilon_{0}>0$ and a function $\delta: \mathbb{R} \rightarrow \mathbb{R}$ with $\lim _{\alpha \rightarrow 0} \delta(\alpha)=0$, such that for all $\varepsilon \in\left(0, \varepsilon_{0}\right]$, all $l \geq l_{0}(\varepsilon / 2)$, all $\eta \in(0,1)$ and all $x_{0} \in D_{c}(l)$ the trajectory $x_{i}$ generated by

$$
x_{i+1}=f\left(x_{i}, u_{\mathcal{P}(l)}\left(x_{i}\right)\right)
$$


satisfies

$$
V\left(x_{i}\right) \leq \max \left\{V\left(x_{0}\right)-(1-\eta) \sum_{j=0}^{i-1} g\left(x_{j}, u_{\mathcal{P}(\ell)}\left(x_{j}\right)\right), \quad \delta(\varepsilon / \eta)+\varepsilon\right\} .
$$

Proof: For each $x \in D$ we define $g_{0}(x):=\min _{u \in \mathcal{U}(x)}\{g(x, u)\}$ and choose $\varepsilon_{0}>0$, such that the implication

$$
g_{0}(x)<\varepsilon_{0} \Rightarrow V(x) \leq c-\varepsilon_{0} / 2
$$

holds. Note that this $\varepsilon_{0}$ exists since $V$ is continuous in a neighborhood of 0 and satisfies $V(0)=0$. We show that our assertion holds for all $\varepsilon \in\left(0, \varepsilon_{0}\right]$ and fix an arbitrary $\varepsilon \in\left(0, \varepsilon_{0}\right]$ and an arbitrary $l \geq l_{0}(\varepsilon / 2)$.

We first show that the implication $x_{i} \in D_{c}(l) \Rightarrow x_{i+1} \in D_{c}(l)$ holds. Let $x \in D_{c}(l)$. Then we obtain

$$
\begin{aligned}
V_{\mathcal{P}}(x)+\varepsilon / 2 \geq V(x) & =\inf _{u \in \mathcal{U}(x)}\{g(x, u)+V(f(x, u))\} \\
& \geq \min _{u \in \mathcal{U}(x)}\left\{g(x, u)+V_{\mathcal{P}(l)}(f(x, u))\right\} \\
& =g\left(x, u_{\mathcal{P}(l)}(x)\right)+V_{\mathcal{P}(l)}\left(f\left(x, u_{\mathcal{P}(l)}(x)\right)\right)
\end{aligned}
$$

which implies

$$
V_{\mathcal{P}(l)}\left(x_{i+1}\right) \leq V\left(x_{i}\right)-g\left(x_{i}, u_{\mathcal{P}(l)}\left(x_{i}\right)\right) \leq V_{\mathcal{P}}\left(x_{i}\right)-g\left(x_{i}, u_{\mathcal{P}(l)}\left(x_{i}\right)\right)+\varepsilon / 2
$$

for all $x_{i} \in D_{c}(l)$. We distinguish two cases:

(a): If $g\left(x_{i}, u_{\mathcal{P}(l)}\left(x_{i}\right)\right) \geq \varepsilon / 2$, then we obtain $V_{\mathcal{P}(l)}\left(x_{i+1}\right) \leq V_{\mathcal{P}}\left(x_{i}\right) \leq c$, i.e., $x_{i+1} \in D_{c}(l)$.

(b): If $g\left(x_{i}, u_{\mathcal{P}(l)}\left(x_{i}\right)<\varepsilon / 2\right.$ then we know $g_{0}\left(x_{i}\right)<\varepsilon / 2 \leq \varepsilon_{0} / 2$, which implies $V\left(x_{i}\right) \leq c-\varepsilon_{0} / 2 \leq c-\varepsilon / 2$ and thus $V_{\mathcal{P}(l)}\left(x_{i+1}\right) \leq V\left(x_{i}\right)+\varepsilon / 2 \leq c$, i.e., again $x_{i+1} \in D_{c}(l)$.

Now for each $x_{i} \in D_{c}(l)$ we know $x_{i+1} \in D_{c}(l) \subseteq D$, thus any trajectory with $x_{0} \in D_{c}(l)$ remains in $D_{c}(l)$ for all future times. Furthermore, since $D_{c}(l) \subseteq D$, for each trajectory starting in $D_{c}(l)$ and all $i \geq 0$ we obtain from (6) the inequality

$$
\begin{aligned}
V\left(x_{i+1}\right) & \leq V_{\mathcal{P}(l)}\left(x_{i+1}\right)+\varepsilon / 2 \\
& \leq V_{\mathcal{P}(l)}\left(x_{i}\right)-g\left(x_{i}, u_{\mathcal{P}(l)}\left(x_{i}\right)\right)+\varepsilon \\
& \leq V\left(x_{i}\right)-g\left(x_{i}, u_{\mathcal{P}(l)}\left(x_{i}\right)\right)+\varepsilon .
\end{aligned}
$$


Now we construct the value $\delta(\alpha)$. We define the set

$$
C_{\alpha}:=\left\{x \in D_{c}(l) \mid g_{0}(x) \leq \alpha\right\}
$$

and set

$$
\delta(\alpha):=\sup _{x \in C_{\alpha}} V(x) .
$$

Note that $\delta(\alpha) \rightarrow 0$ as $\alpha \rightarrow 0$ because $C_{\alpha}$ shrinks down to 0 and $V$ is continuous around 0 with $V(0)=0$. We claim that the implication

$$
V\left(x_{i}\right) \leq \delta(\alpha)+\varepsilon \Rightarrow V\left(x_{i+1}\right) \leq \delta(\alpha)+\varepsilon
$$

holds for all $\alpha \geq \varepsilon$. Again, we distinguish two cases:

(a) $V\left(x_{i}\right) \leq \delta(\alpha)$ : In this case, inequality (8) implies

$$
V\left(x_{i+1}\right) \leq V\left(x_{i}\right)-g\left(x_{i}, u_{\mathcal{P}(l)}\left(x_{i}\right)\right)+\varepsilon \leq V\left(x_{i}\right)+\varepsilon \leq \delta(\alpha)+\varepsilon
$$

which proves (8) in this case.

(b) $V\left(x_{i}\right) \in[\delta(\alpha), \delta(\alpha)+\varepsilon]$ : In this case we know that $x_{i} \notin C_{\alpha}$, hence again using (8) we obtain

$$
V\left(x_{i+1}\right) \leq V\left(x_{i}\right)-\underbrace{g\left(x_{i}, u_{\mathcal{P}(l)}\left(x_{i}\right)\right)}_{\geq \alpha \geq \varepsilon}+\varepsilon \leq V\left(x_{i}\right) \leq \delta(\alpha)+\varepsilon
$$

which also proves invariance.

Finally we show the assertion of the theorem. Fix $\eta \in(0,1)$ and consider an arbitrary $i \in \mathbb{N}_{0}$. If $V\left(x_{i}\right) \leq \delta(\varepsilon / \eta)+\varepsilon$ then we have nothing to show.

If $V\left(x_{i}\right)>\delta(\varepsilon / \eta)+\varepsilon$ then by (8) we can conclude that $V\left(x_{j}\right)>\delta(\varepsilon / \eta)+\varepsilon$ for $j=0, \ldots, i$, thus in particular $x_{j} \notin C_{\varepsilon / \eta}$, i.e., $g\left(x_{j}, u_{\mathcal{P}(l)}\left(x_{j}\right)\right) \geq \varepsilon / \eta$. Hence (8) implies for $j=0, \ldots, i-1$

$$
V\left(x_{j+1}\right) \leq V\left(x_{j}\right)-g\left(x_{i}, u_{\mathcal{P}(l)}\left(x_{i}\right)\right)+\varepsilon \leq V\left(x_{j}\right)-(1-\eta) g\left(x_{i}, u_{\mathcal{P}(l)}\left(x_{i}\right)\right),
$$

because $g\left(x_{i}, u_{\mathcal{P}(l)}\left(x_{i}\right)\right) \geq \varepsilon / \eta$ implies $\varepsilon \leq \eta g\left(x_{i}, u_{\mathcal{P}(l)}\left(x_{i}\right)\right)$.

Applying this inequality iteratively for $j=0, \ldots, i-1$ we obtain

$$
V\left(x_{i}\right) \leq V\left(x_{0}\right)-(1-\eta) \sum_{j=0}^{i-1} g\left(x_{i}, u_{\mathcal{P}(l)}\left(x_{i}\right),\right.
$$

i.e., the assertion. 


\section{Error estimation, adaptive partitions and stabilizing feedback}

The a priori estimate in Theorem 2 has its merits because it shows that and in which sense our feedback definition leads to an approximate optimal performance. From a practical point of view, however, the theorem does not give much information about the structure of the partition $\mathcal{P}$ which is needed in order to achieve a desired level of accuracy.

In particular, our set valued approach is most efficient if we do not use a uniform partition $\mathcal{P}$ but use small cells $P$ only in those regions where a high resolution is really needed. In order to identify such regions we make use of an a posteriori error estimate which is motivated by a similar construction for discrete Hamilton-Jacobi-Bellman equations Grüne (1997), which in turn is based on similar concepts for the numerical solution of partial differential equations.

To this end let $S_{0}=\{x \in X: V(x)<\infty\}$. For $x \in S_{0}$ consider the error function

$$
e(x)=\min _{u \in U}\left\{g(x, u)+V_{\mathcal{P}}(f(x, u))\right\}-V_{\mathcal{P}}(x) .
$$

Note that by definition of $V_{\mathcal{P}}$ we have $e(x) \geq 0$. Since

$$
\begin{aligned}
V(x)-V_{\mathcal{P}}(x) & =\min _{u \in U}\{g(x, u)+V(f(x, u))\}-V_{\mathcal{P}}(x) \\
& \geq \min _{u \in U}\left\{g(x, u)+V_{\mathcal{P}}(f(x, u))\right\}-V_{\mathcal{P}}(x)=e(x),
\end{aligned}
$$

we get

Proposition 3 The error function $e: S_{0} \rightarrow[0, \infty)$ is a lower bound on the error between the true value function $V$ and its approximation $V_{\mathcal{P}}$ :

$$
e(x) \leq V(x)-V_{\mathcal{P}}(x), \quad x \in S_{0}
$$

On a given collection $\mathcal{P}$ with $|\mathcal{P}| \subset S_{0}$ define the error function $e: \mathcal{P} \rightarrow[0, \infty)$ by

$$
e(P)=\max _{x \in P} e(x), \quad P \in \mathcal{P} .
$$

Finally, let $e(\mathcal{P})=\max _{P \in \mathcal{P}} e(P)$.

It should be noted that according to Proposition 3 the error estimate provides a lower bound for the real error but no upper bound; we will illustrate this fact in our numerical examples. Nevertheless, when we look at the performance of the derived feedback law $u_{\mathcal{P}}$ in the sense of Theorem 2 then we see that the error function $e(x)$ does give an upper bound for the error, as shown in the following theorem. 
For its formulation we need a function $\delta$, which for sublevel sets $D_{c}=V_{\mathcal{P}}^{-1}([0, c])$ is defined by

$$
\delta(\varepsilon):=\sup _{x \in C_{\varepsilon}} V(x),
$$

where $C_{\varepsilon}:=\left\{x \in D_{c} \mid g_{0}(x) \leq \varepsilon\right\}$ and $g_{0}(x):=\inf _{u \in \mathcal{U}(x)} g(x, u)$. Note that $\delta(\varepsilon) \rightarrow 0$ as $\varepsilon \rightarrow 0$ because $C_{\varepsilon}$ shrinks down to 0 and $V$ is continuous around 0 with $V(0)=0$.

Theorem 4 Consider a partition $\mathcal{P}$ and a sublevel set $D_{c}=V_{\mathcal{P}}^{-1}([0, c])$ for some $c>0$. Assume that the error estimate e satisfies

$$
e(x) \leq \max \left\{\eta g_{0}(x), \varepsilon\right\}
$$

for all $x \in D_{c}$, some $\varepsilon>0$ and some $\eta \in(0,1)$.

Then the trajectory $x_{i}$ generated by

$$
x_{i+1}=f\left(x_{i}, u_{\mathcal{P}}\left(x_{i}\right)\right)
$$

for each $x_{0} \in D_{c}$ satisfies

$$
V_{\mathcal{P}}\left(x_{i}\right) \leq \max \left\{V_{\mathcal{P}}\left(x_{0}\right)-(1-\eta) \sum_{j=0}^{i-1} g\left(x_{i}, u_{\mathcal{P}(l)}\left(x_{i}\right)\right), \delta(\varepsilon / \eta)+\varepsilon\right\} .
$$

Proof. If $V_{\mathcal{P}}(x) \leq \delta(\varepsilon / \eta)+\varepsilon$ for all $x \in D_{c}$ then there is nothing to show. Thus we can assume $V_{\mathcal{P}}(x)>\delta(\varepsilon / \eta)+\varepsilon$ for some $x \in D_{c}$, i.e., $c>\delta(\varepsilon / \eta)+\varepsilon$. Note that from the construction of $\delta$ the implication

$$
g_{0}(x) \leq \alpha \Rightarrow V(x) \leq \delta(\alpha) \Rightarrow V_{\mathcal{P}}(x) \leq \delta(\alpha)
$$

follows for each $\alpha \geq 0$.

We first show that the implication $x_{i} \in D_{c} \Rightarrow x_{i+1} \in D_{c}$ holds. Let $x_{i} \in D_{c}$. From the assumption of the error estimate we obtain

$$
V_{\mathcal{P}}\left(x_{i+1}\right) \leq V_{\mathcal{P}}\left(x_{i}\right)-g\left(x_{i}, u_{\mathcal{P}}\left(x_{i}\right)\right)+e\left(x_{i}\right) .
$$

for all $x_{i} \in D_{c}$. We distinguish two cases:

(a): If $g\left(x_{i}, u_{\mathcal{P}}\left(x_{i}\right)\right) \geq e(x)$, then we obtain $V_{\mathcal{P}(l)}\left(x_{i+1}\right) \leq V_{\mathcal{P}}\left(x_{i}\right) \leq c$, i.e., $x_{i+1} \in D_{c}$.

(b): If $g\left(x_{i}, u_{\mathcal{P}}\left(x_{i}\right)\right)<e(x)$ then we know $\eta g_{0}\left(x_{i}\right)<e(x)$, thus $e(x)<\varepsilon$ and consequently $g_{0}\left(x_{i}\right)<\varepsilon / \eta$. This implies $V_{\mathcal{P}}\left(x_{i}\right) \leq \delta(\varepsilon / \eta)$ and thus

$$
V_{\mathcal{P}}\left(x_{i+1}\right) \leq V_{\mathcal{P}}\left(x_{i}\right)+\varepsilon \leq \delta(\varepsilon / \eta)+\varepsilon \leq c,
$$


i.e., again $x_{i+1} \in D_{c}$.

Next we show that the implication

$$
V_{\mathcal{P}}\left(x_{i}\right) \leq \delta(\varepsilon / \eta)+\varepsilon \Rightarrow V_{\mathcal{P}}\left(x_{i+1}\right) \leq \delta(\varepsilon / \eta)+\varepsilon
$$

holds. If $e\left(x_{i}\right) \leq \eta g_{0}\left(x_{i}\right)$ then from (13) we immediately obtain $V_{\mathcal{P}}\left(x_{i}\right) \leq$ $V_{\mathcal{P}}\left(x_{i+1}\right)$. It remains to consider $e\left(x_{i}\right)>\eta g_{0}\left(x_{i}\right)$, in which case $e\left(x_{i}\right) \leq \varepsilon$ holds. Again, we distinguish two cases:

(a) $V_{\mathcal{P}}\left(x_{i}\right) \leq \delta(\varepsilon / \eta)$ : In this case, inequality (13) implies

$$
V_{\mathcal{P}}\left(x_{i+1}\right) \leq V_{\mathcal{P}}\left(x_{i}\right)-g\left(x_{i}, u_{\mathcal{P}}\left(x_{i}\right)\right)+\varepsilon \leq V_{\mathcal{P}}\left(x_{i}\right)+\varepsilon \leq \delta(\varepsilon / \eta)+\varepsilon
$$

which proves (14) in this case.

(b) $V\left(x_{i}\right) \in[\delta(\varepsilon / \eta), \delta(\varepsilon / \eta)+\varepsilon]$ : In this case we know that $x_{i} \notin C_{\varepsilon / \eta}$ with $C$. being the set from the definition of $\delta$. Hence again using (13) we obtain

$$
V_{\mathcal{P}}\left(x_{i+1}\right) \leq V_{\mathcal{P}}\left(x_{i}\right)-\underbrace{g\left(x_{i}, u_{\mathcal{P}(l)}\left(x_{i}\right)\right)}_{\geq \varepsilon / \eta \geq \varepsilon}+\varepsilon \leq V_{\mathcal{P}}\left(x_{i}\right) \leq \delta(\varepsilon / \eta)+\varepsilon
$$

which also proves the invariance (14).

Finally we show the assertion of the theorem. Consider an arbitrary $i \in \mathbb{N}_{0}$. If $V\left(x_{i}\right) \leq \delta(\varepsilon / \eta)+\varepsilon$ then we have nothing to show.

If $V_{\mathcal{P}}\left(x_{i}\right)>\delta(\varepsilon / \eta)+\varepsilon$ then by (14) we can conclude that $V_{\mathcal{P}}\left(x_{j}\right)>\delta(\varepsilon / \eta)+\varepsilon$ for $j=0, \ldots, i$, thus also $V\left(x_{j}\right)>\delta(\varepsilon / \eta)+\varepsilon$ and in particular $x_{j} \notin C_{\varepsilon / \eta}$, i.e., $g\left(x_{j}, u_{\mathcal{P}(l)}\left(x_{j}\right)\right) \geq \varepsilon / \eta$ which implies $e(x) \leq \eta g\left(x_{j}, u_{c P(l)}\left(x_{j}\right)\right)$. Hence $(8)$ implies for $j=0, \ldots, i-1$

$V_{\mathcal{P}}\left(x_{j+1}\right) \leq V_{\mathcal{P}}\left(x_{j}\right)-g\left(x_{i}, u_{\mathcal{P}(l)}\left(x_{i}\right)\right)+e\left(x_{i}\right) \leq V_{\mathcal{P}}\left(x_{j}\right)-(1-\eta) g\left(x_{i}, u_{\mathcal{P}(l)}\left(x_{i}\right)\right)$.

Applying this inequality iteratively for $j=0, \ldots, i-1$ we obtain

$$
V_{\mathcal{P}}\left(x_{i}\right) \leq V_{\mathcal{P}}\left(x_{0}\right)-(1-\eta) \sum_{j=0}^{i-1} g\left(x_{i}, u_{\mathcal{P}}\left(x_{i}\right),\right.
$$

i.e., the assertion.

Note that the inequality (10) makes use of two "tuning" parameters, namely $\eta$ and $\varepsilon$. For each fixed $\eta \in(0,1)$, the parameter $\varepsilon>0$ induces an upper bound for the lowest achievable value of $V_{\mathcal{P}}\left(x_{i}\right)$, while $\eta \in(0,1)$ controls the speed of decay of $V_{\mathcal{P}}\left(x_{i}\right)$ until the lowest value is reached. Clearly, when aiming at an approximately optimal feedback whose performance is as good as possible, one 
would choose $\eta<<1$ and $\varepsilon / \eta<<1$. This, in turn, requires a very fine partition which poses considerably numerical problems both in terms of computation time and memory consumption, especially in higher dimensions, because, like in traditional dynamic programming schemes, out procedure is subject to the curse of dimensionality.

Keeping in mind that the main purpose of the kind of optimal control problems treated in our setup is the derivation of asymptotically stabilizing feedback laws one might therefore ask to relax the strict "approximate optimality" condition by looking only for a feedback which - although far from optimal still ensures approximate asymptotic stability in a suitable sense. Taking into account the nature of the two parameters, in terms of asymptotic stability the parameter $\varepsilon$ describes the size of a neighborhood of the origin where every trajectory starting in $D_{c}$ will eventually end while $\eta$ describes the convergence rate to this neighborhood. Therefore, it may be a good compromise to choose a relatively large $\eta \in(0,1)$. This way we slow down the convergence of the trajectories to the (neighborhood of the) origin, but in turn the problem becomes numerically easier and can be solved on a coarser partition. Such relaxations of the optimality conditions have recently been used also for other dynamic programming formulations of optimal control problems, see Lincoln and Rantzer (2002), and can considerably reduce the computational cost.

We will make this statement precise using a general framework for the derivation of rigorous stability estimates for approximately optimal feedback laws developed in Nešić and Teel (2004). A first application of this framework to numerical optimal control was given in Grüne and Nešić (2003), where the effect of numerical time discretization in sampled data controller design was investigated. Likewise, the framework from Nešić and Teel (2004) allows the conclusion of asymptotic stability in our framework, as stated in the following corollary. The result is stated using the by now standard definitions of comparison functions 1

Corollary 1 Let the assumptions of Theorem 4 be satisfied. Then for any $\eta \in(0,1)$ the feedback law $u_{\mathcal{P}}$ renders the closed loop system

$$
x_{i+1}=f\left(x_{i}, u_{\mathcal{P}}\left(x_{i}\right)\right)
$$

practically asymptotically stable on $D_{c}$, i.e., there exists a $\mathcal{K} \mathcal{L}$-function $\beta$ depending on $g_{0}$ and $\eta$, with the property that for any $\delta>0$ there exists $\varepsilon>0$

$\overline{1}$ As usual we call a function $\alpha$ of class $\mathcal{K}_{\infty}$ if it is a homeomorphism of $[0, \infty)$ (i.e., $\alpha(0)=0$ and $\alpha(r) \rightarrow \infty$ as $r \rightarrow \infty$ ), a continuous function $\beta$ in two real nonnegative arguments is called of class $\mathcal{K} \mathcal{L}$ if it is of class $\mathcal{K}_{\infty}$ in the first and decreasing to zero in the second argument. 
such that the inequality

$$
\left\|x_{i+1}\right\| \leq \beta\left(\left\|x_{0}\right\|, t\right)+\delta
$$

holds for each $x_{0} \in D_{c}$ and all partitions for which the error estimate e satisfies the assumption of Theorem 4 with the given $\varepsilon$.

Proof. From (13) we obtain the inequality

$$
V_{\mathcal{P}}\left(x_{i+1}\right) \leq V_{\mathcal{P}}\left(x_{i}\right)-\eta g_{0}\left(x_{i}\right)+\varepsilon
$$

This estimate implies that on $D_{c}$ all assumptions of (Nešić and Teel, 2004, Theorem 1) are satisfied except the continuity of $V_{\mathcal{P}}$. An inspection of the proof of (Nešić and Teel, 2004, Theorem 1), however, reveals that continuity of $V$ is only needed if the feedback was designed using an approximation of $f$ which is not the case in our setting.

It is worth noting that even though continuity of $V_{\mathcal{P}}$ is not needed in the proof of the previous corollary, in general one cannot expect robustness of the feedback law even for arbitrarily small perturbations $\tilde{f}$ of $f$ if the controller design is based on the discontinuous (Lyapunov) function $V_{\mathcal{P}}$. In practice, small perturbations of $f$ can only cause problems if the image $f\left(x, u_{\mathcal{P}}(x)\right)$ lies close to the boundary of a cell, because as long as the perturbed image $\tilde{f}\left(x, u_{\mathcal{P}}(x)\right)$ lies in the same cell as $f\left(x, u_{\mathcal{P}}(x)\right)$, the inequality (13) remains valid. Thus, we can expect that in general the feedback should behave rather robust.

Nevertheless, in order to obtain rigorous robustness results our construction of $V_{\mathcal{P}}$ and $u_{\mathcal{P}}$ needs to be modified. For instance, the computation of $V_{\mathcal{P}}$ could be based on a set valued "inflation" $f+B_{\varepsilon}(0)$ instead of $f$, an idea which bears some similarity with the rigorous discretization methods for set valued numerical methods presented in Junge (2000a b) (see also Grüne, 2002, Chapter 5)). In order to keep the presentation simple, we will not go into details here and address this question in a later paper.

\section{$6 \quad$ Numerical examples}

We are going to illustrate the results of the previous sections by means of two example computations: first we consider a very simple control system with a one-dimensional state space for which we can compute all quantities explicitely. As the second example we choose a controlled pendulum that is supposed to be steered into the ("upper") unstable equilibrium. 


\subsection{A simple $1 D$ system}

Consider the system

$$
x_{k+1}=x_{k}+(1-a) u_{k} x_{k}, \quad k=0,1, \ldots,
$$

where $x_{k} \in X=[0,1], u_{k} \in U=[-1,1]$ and $a \in(0,1)$ is a fixed parameter. As the instantaneous cost function we choose

$$
g(x, u)=(1-a) x
$$

such that the optimal control policy is to steer to the origin as fast as possible, i.e. for every $x$, the optimal control policy is $\mathbf{u}(x)=(-1,-1, \ldots)$. This yields $V(x)=x$ as the optimal value function.

For the following computations we consider the value $a=0.8$ and a partition $\mathcal{P}$ of $[0,1]$ into 64 intervals. The weights on the edges of the graph (2) are approximated by minimizing (3) over 100 equally spaced test points in each interval $P \in \mathcal{P}$ and 10 equidistant points in $U$.

Figure 1 (left) shows $V$ (red line) and the approximate optimal value function $V_{\mathcal{P}}$ (blue bars), as well as (right) the true error $E(P)=\max _{x \in P} V(x)-V_{\mathcal{P}}(x)$, $P \in \mathcal{P}$, (blue bars) and the error function $e: \mathcal{P} \rightarrow[0, \infty)$ (red bars).
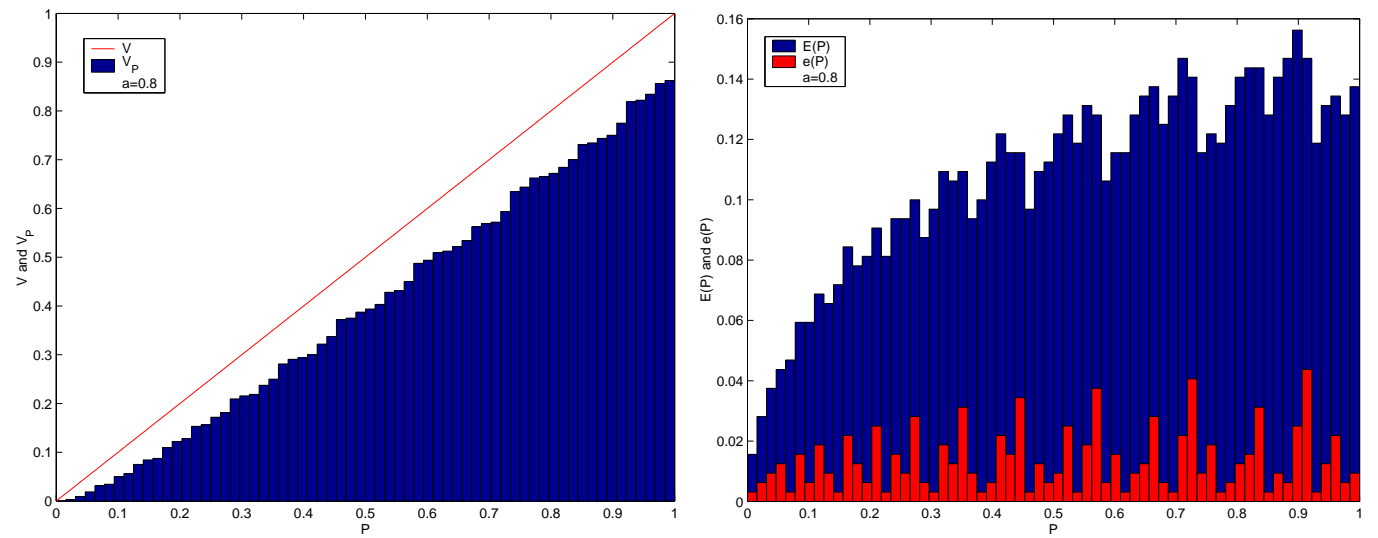

Fig. 1. Left: true (red) and approximate (blue) optimal value function. Right: true error $E(P)$ (blue) and error function $e(P)$ (red) ( $a=0.8$, equipartition of 64 intervals).

We note that $D_{1}=X=[0,1], g_{0}(x)=\min _{u \in U} g(x, u)=(1-a) x$, and thus $C_{\varepsilon}=\{x \in[0,1] \mid x \leq \varepsilon /(1-a)\}$, as well as $\delta(\varepsilon)=\varepsilon /(1-a)$. Using these formulas we can compare the value of $V_{\mathcal{P}}$ to the estimate (12) for different choices of $\eta$ (we choose $\varepsilon=\max \left\{e(x) \mid e(x)>\eta g_{0}(x)\right\}$ ). Figure 2 shows $V_{\mathcal{P}}$ and the left hand side of (12) for four different values of $\eta$ along a feedback trajectory to the initial point $x=1$. Note that in this example the cost function $g$ does actually not depend on the control. In the computation of the 


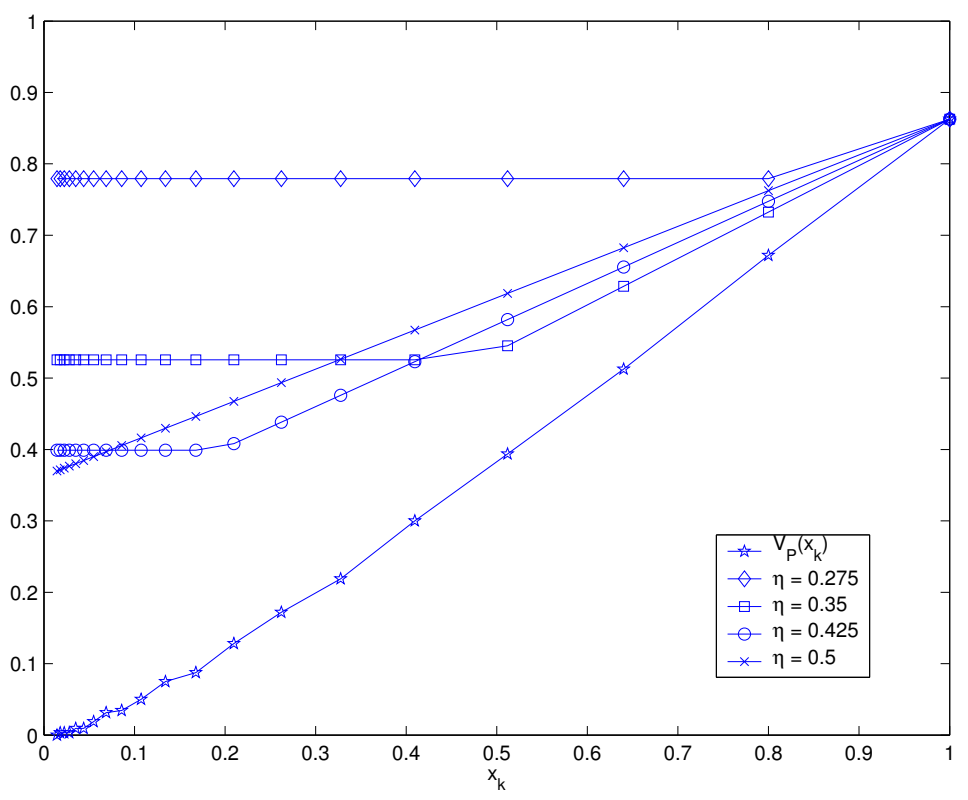

Fig. 2. Comparison of $V_{\mathcal{P}}$ and the estimate (12) for different values of $\eta$. The markers indicate the values at points $x_{k}$ of a trajectory generated by (11).

feedback control in $(5)$ this leads to $u_{\mathcal{P}}(x)$ being not unique. For the above computation we have chosen $u_{k}=-1$. A different choice may actually lead to a non-stabilizing control policy: note that for $u_{k}=0$ every point in $[0,1]$ is a fixed point of (15).

\subsection{Inverted pendulum}

As the second example, we reconsider a computation from Junge and Osinga (2004):

$$
\begin{aligned}
& \dot{x}_{1}=x_{2} \\
& \dot{x}_{2}=\frac{\frac{g}{l} \sin \left(x_{1}\right)-\frac{1}{2} m_{r} x_{2}^{2} \sin \left(2 x_{1}\right)-\frac{m_{r}}{m l} \cos \left(x_{1}\right) u}{\frac{4}{3}-m_{r} \cos ^{2}\left(x_{1}\right)}
\end{aligned}
$$

The equations model the motion of a (planar) inverted pendulum on a cart which moves under an applied horizontal force $u$. The position $x_{1}$ of the pendulum is measured relative to the position of the cart as the offset angle from the vertical up position. The parameters are as in Junge and Osinga (2004), $M=8, m=2, m_{r}=m /(m+M), l=0.5, g=9.8$. The instantaneous cost is

$$
q(x, u)=\frac{1}{2}\left(0.1 x_{1}^{2}+0.05 x_{2}^{2}+0.01 u^{2}\right)
$$



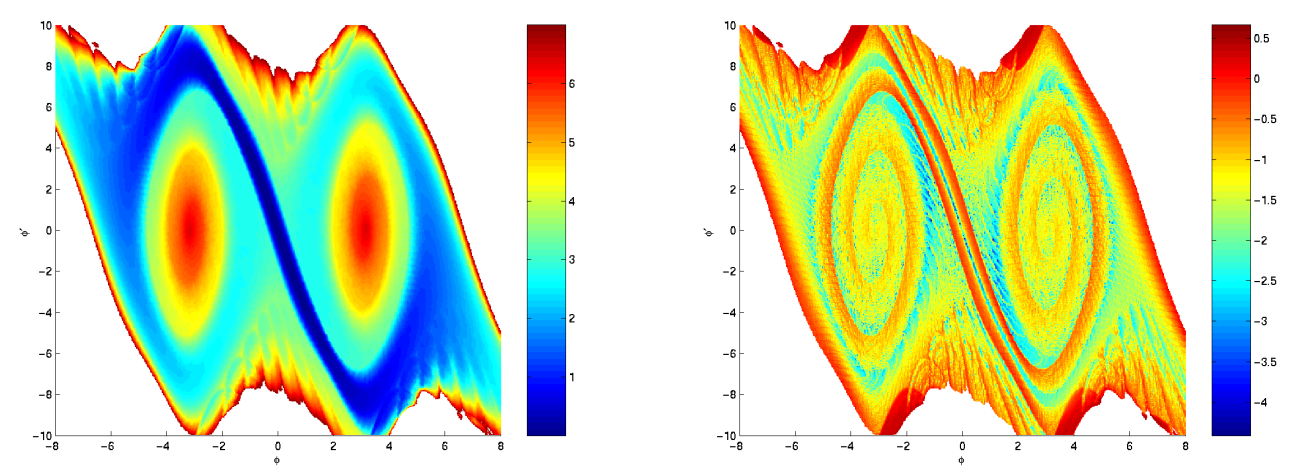

Fig. 3. Approximate optimal value function (left) and local error indicator (right, logarithmic scaling of the coloring) for $V_{\mathcal{P}}^{-1}([0,7])$ on depth 18 .

Considering the time- $T$-map of this system (with $T=0.1$, computed via the Runge-Kutta scheme of 4 -th order with step size 0.02 ), we arrive at the cost function

$$
g(x, u)=\int_{0}^{T} q\left(\phi^{t}(x ; u), u\right) d t
$$

where $u$ denotes the constant function with value $u$ on $[0, T]$. We choose $X=$ $[-8,8] \times[-10,10]$ as the region of interest, partitioned into $2^{18}$ boxes of size $1 / 32 \times 5 / 128$. For the computation of the weights (3) we used the vertices of each box in phase space and the set $\{-64,-56, \ldots,-8,0,8, \ldots, 56,64\}$ for $U$.

Figure 3 shows the approximate optimal value function $V_{\mathcal{P}}$ as computed on this partition as well as the local error indicator (9). In Figure 4 we compare the feedback trajectory as generated by (11) with a pseudo-trajectory (cf. the discussion at the beginning of Section 4) obtained by following the shortest path in the graph (2) in phase space, while in Figure 5 we visualize their time-dependence, together with the piecewise constant approximate optimal control policy.

\section{References}

Dellnitz, M., Hohmann, A., 1997. A subdivision algorithm for the computation of unstable manifolds and global attractors. Numer. Math. 75 (3), 293-317.

Dellnitz, M., Junge, O., 2002. Set oriented numerical methods for dynamical systems. In: Fiedler, B. (Ed.), Handbook of Dynamical Systems. Volume 2. Elsevier, Amsterdam, pp. 221-264.

Dijkstra, E., 1959. A note on two problems in connection with graphs. Numer. Math. 5, 269-271.

Fontes, F. A. C. C., 2001. A general framework to design stabilizing nonlinear model predictive controllers. Syst. Contr. Lett. 42, 127-143.

Freeman, R. A., Kokotovic, P. V., 1996. Inverse optimality in robust stabilization. SIAM J. Control Optim. 34 (4), 1365-1391. 


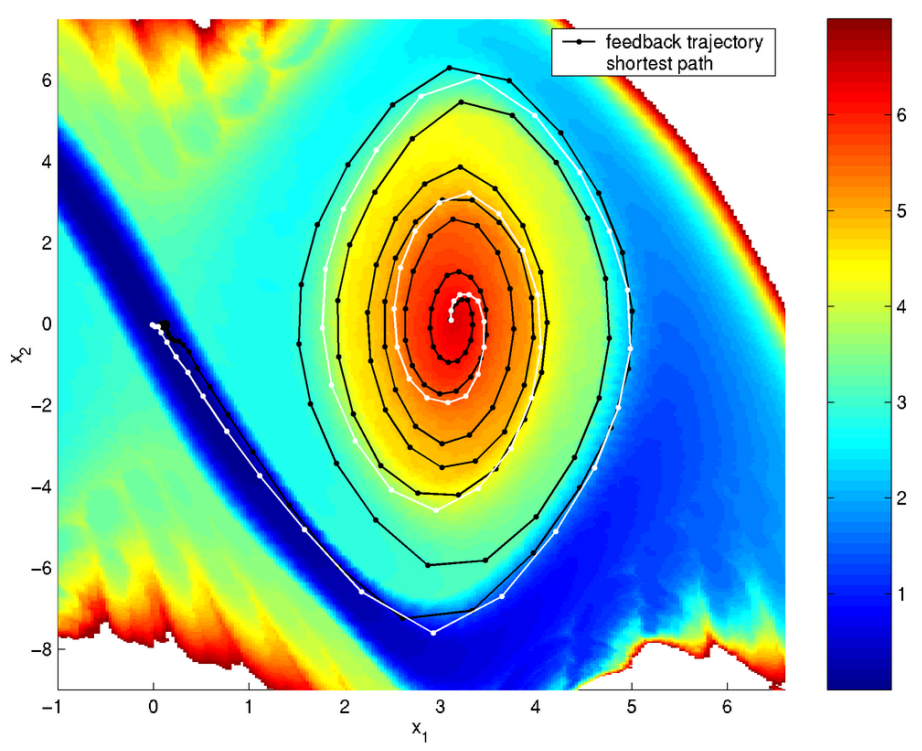

Fig. 4. Pseudo-trajectory as given by the shortest path (white) and optimal feedback trajectory (black) for the initial point $(3.1,0.1)$.
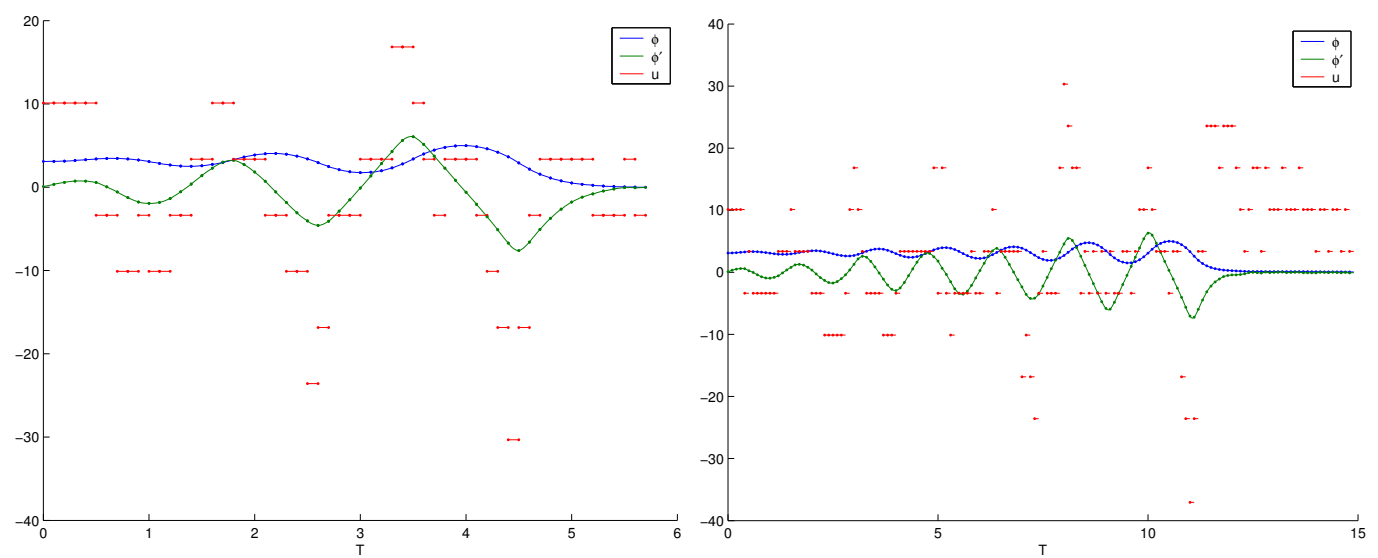

Fig. 5. Pseudo-trajectory as given by the shortest path (left) and optimal feedback trajectory (right) for the initial point $(3.1,0.1)$. The corresponding approximate optimal control function is shown in red, respectively.

Freeman, R. A., Kokotović, P. V., 1996. Robust nonlinear control design. Systems \& Control: Foundations \& Applications. Birkhäuser Boston Inc., Boston, MA, state-space and Lyapunov techniques.

Grüne, L., 1997. An adaptive grid scheme for the discrete Hamilton-JacobiBellman equation. Numer. Math. 75 (3), 319-337.

Grüne, L., 2000. Homogeneous state feedback stabilization of homogeneous systems. SIAM J. Control Optim. 38, 1288-1314.

Grüne, L., 2002. Asymptotic Behavior of Dynamical and Control Systems under Perturbation and Discretization. Lecture Notes in Mathematics, Vol. 1783. Springer-Verlag. 
Grüne, L., Nešić, D., 2003. Optimization based stabilization of sampled-data nonlinear systems via their approximate discrete-time models. SIAM J. Control Optim. 42, 98-122.

Grüne, L., Wirth, F., 1999. Feedback stabilization of discrete-time homogeneous semi-linear systems. Syst. Contr. Lett. 37, 19-30.

Junge, O., 2000a. Mengenorientierte Methoden zur numerischen Analyse dynamischer Systeme. Shaker Verlag, Aachen, dissertation, Universität Paderborn.

Junge, O., 2000b. Rigorous discretization of subdivision techniques. In: Fiedler, B., Gröger, K., Sprekels, J. (Eds.), EQUADIFF 99, Proceedings of the International Congress held in Berlin, Germany. World Scientific, Singapore, pp. 916-918.

Junge, O., Osinga, H., 2004. A set oriented approach to global optimal control. ESAIM: Control Optim. Calc. Var.To appear.

Kreisselmeier, G., Birkhölzer, T., 1994. Numerical nonlinear regulator design. IEEE Trans. Autom. Control 39 (1), 33-46.

Lincoln, B., Rantzer, A., 2002. Suboptimal dynamic programming with error bounds. In: Proceedings of the 41th IEEE Conference on Decision and Control, Las Vegas, NV, USA. pp. 2354-2359.

Mayne, D. Q., Rawlings, J. B., Rao, C. V., Scokaert, P. O. M., 2000. Constrained model predictive control: stability and optimality. Automatica 36, 789-814.

Nešić, D., Teel, A. R., 2004. A framework for stabilization of nonlinear sampled-data systems based on their approximate discrete-time models. IEEE Trans. Autom. ControlTo appear.

Sontag, E. D., 1998. Mathematical Control Theory, 2nd Edition. Springer Verlag, New York.

Szolnoki, D., 2003. Set oriented methods for computing reachable sets and control sets. Discrete Contin. Dyn. Syst. Ser. B 3 (3), 361-382. 